\title{
COMPOSITION DU PEUPLEMENT PISCICOLE D'UN PETIT ESTUAIRE ANTHROPISÉ (LE COUESNON, FRANCE).
}

\author{
P. LAFFAILLE (1), L. THIEULLE (2), E. FEUNTEUN (1) ET J.C. LEFEUVRE (1).
}

(1) Université de Rennes 1, UMR 6553, Campus Beaulieu, 35042 Rennes Cedex, France. Email : pascal.lafaille@univ-rennes.fr

(2) Fish-Pass, 91 avenue de Saint-Brieuc, 35000 Rennes, France.

\section{RÉSUMÉ}

Dans un petit estuaire du nord de la Bretagne (France), l'ichtyofaune a été échantillonnée lors de chaque saison d'une année sur six stations selon une répartition amont-aval. 28 espèces ont ainsi été capturées à l'aide de deux engins de pêche, une senne de plage couplée à un tramail. Selon les indices d'abondance numériques et pondéraux, trois zones peuvent être identifiées. Une zone estuarienne marine en aval qui est composée essentiellement d'espèces marines et résidentes de la baie adjacente. Le peuplement est dominé par les gobies buhottes et par les juvéniles de plies, de bars et de mulets. La diversité piscicole et les indices d'abondance sont plus élevés que dans les autres zones. Les stations les plus en amont correspondent à la zone estuarienne dulçaquicole qui est presque exclusivement composée d'espèces dulcicoles. Ce peuplement est dominé par les brèmes, les gardons et les chevaines. Mais les indices d'abondance sont les plus faibles à cause de la banalisation et de la chenalisation des berges. La troisième zone correspond à la zone estuarienne intermédiaire. La présence d'espèces d'origine marine et littorale y est limitée par un barrage estuarien. Cet aménagement hydraulique, situé très en aval, réduit ainsi la fonction de nurserie de cet estuaire vis à vis des espèces marines. La répartition spatiale du peuplement piscicole est donc le résultat d'un facteur abiotique (la salinité) et de l'action anthropique (le barrage et la perte des habitats).

Mots-clés : Ichtyofaune, estuaire, variabilité, aménagement hydraulique, barrage estuarien. 


\title{
COMPOSITION OF FISH COMMUNITY IN SMALL ANTHROPIC ESTUARY (THE « COUESNON », FRANCE).
}

\begin{abstract}
In a small estuary of the North Brittany (France), fish communities were sampled during one year at each season on six stations according to a downstream gradient. 28 species were caught by a beach seine coupled with a trammel net. According to numeric and biomass abundance index, three zones were identified. A marine estuarine zone which was composed essentially of marine and resident species of the neighbouring bay. This community was dominated by sand gobies Pomatoschistus minutus and by young flounders, sea bass and mullets. Species richness and abundance index were higher than in the other zones of this estuary. Most upstream station, corresponding to the freshwater estuarine zone, were characterised by the almost exclusive dominance of freshwater species. This community was dominated by breams, roaches and chubs. But abundance index were lowest because of disturbed habitat due to embanking. Presence of marine and littoral species in the intermediate estuarine zone is limited by the presence of a estuarine dam, located at the outlet of the river, which reduces, nursery function of this estuary. Also, spatial distribution of the fish communities is the result of a abiotic factor (salinity) and of anthropic action (the dam and habitat loss).
\end{abstract}

Key-words : fish communities, estuary, variability, hydraulic works, estuarine dam.

\section{INTRODUCTION}

Les estuaires sont des zones de transition entre la mer, les eaux douces et les terres. Cet écotone couplé avec un continuum eau douce - eau salée entraîne une dynamique estuarienne complexe et une forte productivité biologique (MANN, 1982). L'utilisation des estuaires par la faune piscicole comme zone trophique et spécialement comme nurserie (ROGERS et al., 1984 ; COSTA, 1988) a souvent été attribuée à cette forte productivité (MC LUSKY, 1981) bien que d'autres facteurs tels la réduction des taux de salinité et de la prédation aquatique jouent aussi un rôle non négligeable (BLABER et BLABER, 1980 ; WHITFIELD, 1983 ; CLARIDGE et POTTER, 1984). Cette importante fonction vis à vis de nombreuses espèces piscicoles a essentiellement été étudiée dans des habitats marins et dulçaquicoles, et peu d'attention a été portée sur les eaux saumâtres (ROZAS et ODUM, 1987), probablement à cause des difficultés d'échantillonnage. De plus, la plupart des études se sont focalisées sur les grands estuaires et peu de données existent pour des zones de plus faible superficie.

Un des premiers pas dans la connaissance de ces écosystèmes complexes et la compréhension du rôle important qu'ils jouent dans le maintien des stocks côtiers des poissons sont d'identifier les organismes présents, leur distribution et leur abondance relative (CAIN et DEAN, 1976 ; COSTA, 1989). Ce travail présente les premières données sur la répartition spatio-temporelle du peuplement piscicole d'un petit estuaire breton, typique de nombreux estuaires européens, où les activités humaines ont eu un profond impact. 


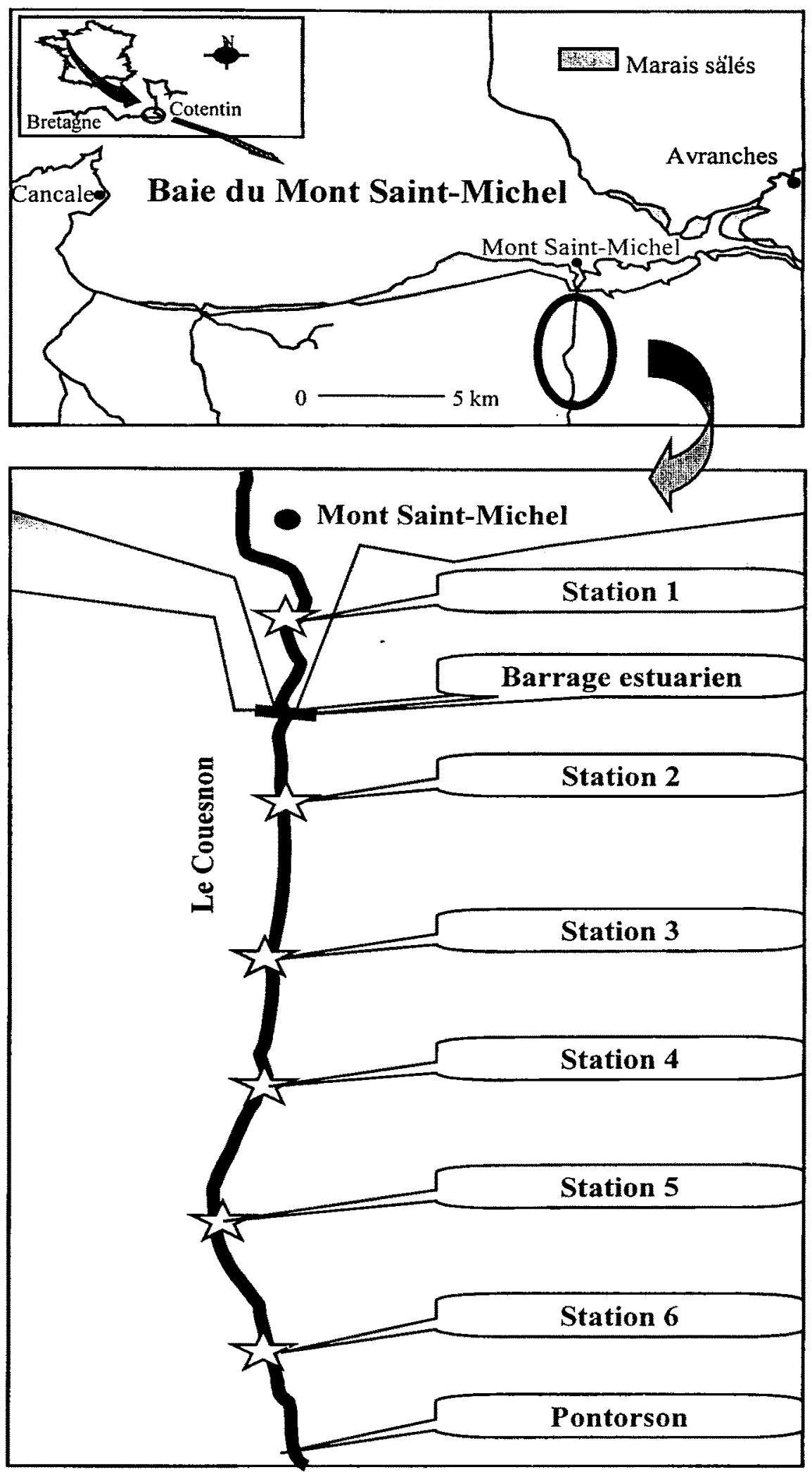

Figure 1

Localisation de l'estuaire du Couesnon et des sites d'études.

Figure 1

Location of Couesnon estuary and study sites. 


\section{Matériel et méthodes}

\section{Site d'étude}

Le Couesnon est un petit fleuve côtier (bassin versant d'environ $1120 \mathrm{~km}^{2}$ ) situé entre la Bretagne et la Normandie (France) et rejoignant la mer au niveau du Mont SaintMichel dans la baie du même nom (Figure 1). Un barrage estuarien (40 $\mathrm{m}$ de long) constitué de portes à flot passif contrôle les apports des marées dans le Couesnon. Dans cet estuaire les débits mensuels moyens varient entre $2.2 \mathrm{~m}^{3} \cdot \mathrm{s}^{-1}$ lors des étiages et $22.5 \mathrm{~m}^{3} \cdot \mathrm{s}^{-1}$ lors des crues (DANAIS et al., 1987).

Lichtyofaune a été échantillonnée dans 6 stations selon une répartition amont-aval. Dans ces stations le substrat est vaseux et les rives souvent chenalisées et stabilisées par des blocs rocheux. Les hydrophytes sont inexistants et les hélophytes assez rares. La première station, bordée par les marais salés tidaux, est située en aval du barrage et est soumise à l'influence directe des marées. Les stations 2,3 et 4 , situées en amont du barrage, sont constituées d'un plat vaseux, d'une roselière sur une rive et d'une digue rocheuse sur l'autre. Les stations 5 et 6 , les plus en amont, sont situées dans une zone chenalisée ; leurs berges sont abruptes et rocheuses. La station 1 se situe à $1.5 \mathrm{~km}$ en aval du barrage et la station 6 à environ $6.5 \mathrm{~km}$ en amont.

\section{Échantillonnage de l'ichtyofaune}

Lichtyofaune a été échantillonnée une fois par saison (entre mai 1998 et février 1999) dans les six stations par deux engins de pêche : une senne de plage (10 $\mathrm{mm}$ de maille, $25 \mathrm{~m}$ de long, $2 \mathrm{~m}$ de hauteur) et un filet tramail ( 30 et $70 \mathrm{~mm}$ de maille, $25 \mathrm{~m}$ de long, $2 \mathrm{~m}$ de hauteur). Le couplage de ces deux engins aux caractéristiques complémentaires permet d'augmenter l'effort de pêche et de capturer pratiquement toutes les espèces présentes dans l'estuaire. L'avantage de cette méthode est d'obtenir des indices d'abondance plus ou moins standardisés. La technique consiste à repousser avec la senne les gros individus vers le filet maillant qui est plus à même de les capturer. Pour chaque station, un trait de senne par berge et par saison a été réalisé lors d'une année. Chaque poisson capturé a été identifié, mesuré (longueur à la fourche au $\mathrm{mm}$ près) et pesé à $0.5 \mathrm{~g}$ près. Les indices numériques et pondéraux ont ainsi pu être estimés en fonction de la surface prospectée avec la senne de plage (250 $\mathrm{m}^{2}$ en moyenne). Les variations spatio-temporelles du peuplement ont été analysées par une classification hiérarchique des indices d'abondance (méthode de Ward, distance euclidienne), après avoir transformé les données en log $(x+1)$ (FIELDS et al., 1982).

Durant cette étude, les températures et les salinités de surface et de fond ont été mesurées pour chaque station lors de chaque campagne de pêche.

\section{RÉSULTATS}

\section{Températures et salinités}

Du point de vue des températures (min. : $4{ }^{\circ} \mathrm{C}$, max. : $24^{\circ} \mathrm{C}$ ) les quatre saisons peuvent être clairement identifiées : de 14 à $18^{\circ} \mathrm{C}$ au printemps, de 20 à $26^{\circ} \mathrm{C}$ en été, de 12 à $13^{\circ} \mathrm{C}$ en automne et de 4 à $7{ }^{\circ} \mathrm{C}$ en hiver (Tableau 1). Au contraire, aucun gradient entre l'amont et l'aval ne peut être observé. Concernant les taux de salinité, c'est durant les campagnes d'été (30\% pour la station 1 et $15 \%$ pour la station 6 ) et de printemps (23\% pour la station 1, $12 \%$ pour la station 4 et $0 \%$ pour la station 6 ) que l'influence de la marée est la plus importante. En automne et en hiver, les salinités sont nulles en amont des portes à flot (respectivement $28 \%$ et $15 \%$ pour la station aval 1 et 0 pour toutes les autres). Avant la construction du barrage estuarien, l'influence des marées pouvait se faire ressentir jusqu'à Pontorson (10 km environ en amont du barrage). 


\section{Tableau I}

Température $\left({ }^{\circ} \mathrm{C}\right)$ et salinité $(\%)$ de fond et de surface des différentes stations. Le barrage estuarien se situe entre les stations 1 et 2.

\section{Table I}

Bottom and surface temperature $\left({ }^{\circ} \mathrm{C}\right)$ and salinity $(\% \circ)$ of different stations. The estuarine dam is situated between stations 1 and 2.

\begin{tabular}{|c|c|c|c|c|c|c|c|c|c|c|c|c|c|}
\hline & \multirow[b]{2}{*}{ Station } & \multicolumn{6}{|c|}{ Température $\left({ }^{\circ} \mathrm{C}\right)$} & \multicolumn{6}{|c|}{ Salinité (\%o) } \\
\hline & & 1 & 2 & 3 & 4 & 5 & 6 & 1 & 2 & 3 & 4 & 5 & 6 \\
\hline & Saison & & & & & & & & & & & & \\
\hline \multirow{4}{*}{ Surface } & Printemps & 18 & 18 & 17 & 17 & 15 & 15 & 23 & 0 & 0 & 0 & 0 & 0 \\
\hline & Eté & 20 & 24 & 26 & 26 & 22 & 23 & 30 & 3 & 3 & 3 & 0 & 0 \\
\hline & Automne & 13 & 13 & 13 & 13 & 13 & 13 & 28 & 0 & 0 & 0 & 0 & 0 \\
\hline & Hiver & 7 & 4 & 4 & 4 & 4 & 4 & 15 & 0 & 0 & 0 & 0 & 0 \\
\hline \multirow[t]{4}{*}{ Fond } & Printemps & 18 & 16 & 16 & 16 & 14 & 14 & 23 & 21 & 22 & 12 & 0 & 0 \\
\hline & Eté & 21 & 22 & 24 & 23 & 21 & 22 & 30 & 21 & 26 & 23 & 21 & 15 \\
\hline & Automne & 13 & 12 & 12 & 12 & 12 & 12 & 28 & 0 & 0 & 0 & 0 & 0 \\
\hline & Hiver & 7 & 4 & 4 & 4 & 4 & 4 & 15 & 0 & 0 & 0 & 0 & 0 \\
\hline
\end{tabular}

\section{Composition du peuplement}

Lors de cette étude, 28 espèces appartenant à 4 groupes écologiques ont été identifiées (Tableau 2). Deux espèces sont des migrateurs amphihalins : l'anguille européenne et le saumon atlantique. Huit espèces sont d'origine marine. Ce sont tous des poissons euryèces supportant les fortes variations de température et de salinité. Six espèces sont résidentes des vasières tidales de la baie adjacente. Ce sont les gobies du genre Pomatoschitus et les syngnathidés. Le dernier groupe, possédant la plus forte richesse spécifique, est formé par les espèces dulçaquicoles.

Ce peuplement, ayant un indice d'abondance moyen de 1250 poissons et $6.6 \mathrm{~kg}$ par hectare, est dominé numériquement par 7 espèces. Quatre espèces représentent près de $54 \%$ des effectifs totaux : le gobie buhotte, la plie, le bar et les alevins de mulet, qui sont d'origine marine ou résidentes de la baje. Les 3 autres espèces, le gardon, la brème commune et le chevaine, sont dulçaquicoles et représentent environ $29 \%$ des effectifs capturés. Ces trois dernières espèces dominent aussi la structure pondérale du peuplement $(\% \mathrm{~B}=54 \%)$. Le gardon ( $\% \mathrm{~F}=63 \%$ ) et la brème commune ( $\% \mathrm{~F}=56 \%)$ sont les deux espèces présentes dans le plus grand nombre d'échantillons. 


\section{Tableau II}

Indices d'abondance moyens des effectifs ( $\mathrm{nb} / \mathrm{ha}$ ) et des biomasses ( $\mathrm{g} / \mathrm{ha}$ ), abondance relative numérique $(\% \mathrm{~N})$ et pondérale $(\% \mathrm{~B})$, et fréquence d'occurrence (\%F) de l'ichtyofaune échantillonnée dans l'estuaire du Couesnon. Classement par \%F décroissant.

\section{Table II}

Mean abundance index of density ( $\mathrm{hb} / \mathrm{ha}$ ) and biomass ( $\mathrm{kg} / \mathrm{ha}$ ), density frequency $(\% \mathrm{~N})$ and biomass frequency $(\% \mathrm{~B})$, and occurrence $(\% \mathrm{~F})$ of fish community sampled in Couesnon estuary. Species are listed in decreasing order of occurrence.

\begin{tabular}{|c|c|c|c|c|c|c|}
\hline Espèces & Noms communs & $\begin{array}{l}\text { Effectif } \\
\text { moyen }\end{array}$ & $\% N$ & $\begin{array}{l}\text { Biomasse } \\
\text { moyenne }\end{array}$ & $\% \mathrm{~B}$ & $\% F$ \\
\hline Rutilus rutilus & Gardon & 138,8 & 11,1 & 832,8 & 12,7 & 63 \\
\hline Abramis brama & Brème commune & 110,7 & 8,9 & 1461,2 & 22,3 & 56 \\
\hline Pleuronectes platessa & Plie & 189,8 & 15,2 & 279,6 & 5,8 & 49 \\
\hline Alburnus alburnus & Ablette & 45,7 & 3,7 & 594,1 & 9,0 & 46 \\
\hline Pomatoschistus minutus & Gobie buhotie & 243,3 & 19,5 & 172,2 & 2,6 & 44 \\
\hline Leuciscus cephalus & Chevaine & 107,3 & 8,6 & 1212,5 & 18,5 & 44 \\
\hline Mugil sp. & Alevin de mulet & 98,7 & 7,9 & 345,5 & 5,3 & 44 \\
\hline Dicentrarchus labrax & Bar & 138,5 & 11,1 & 94,2 & 1,4 & 32 \\
\hline Platichthys hesus & Flet & 61,3 & 4,9 & 459,8 & 7,0 & 32 \\
\hline Anguilla anguilla & Anguille & 8,7 & 0,7 & 330,6 & 5,0 & 32 \\
\hline Pomatoschistus lozanoi & Gobie de Lozanoi & 26,5 & 2,1 & 23,9 & 0,4 & 26 \\
\hline Gasterosteus aculeatus & Epinoche & 12,2 & 1,0 & 8,5 & 0,1 & 25 \\
\hline Blicca bjoerkna & Brème bordelière & 27,7 & 2,2 & 127,4 & 1,9 & 19 \\
\hline Sprattis spratius & Sprat & 22,2 & 1,8 & 5,6 & 0,1 & 11 \\
\hline Scardinius enthrophthalmus & Rotengle & 3,7 & 0,3 & 54,8 & 0,8 & 7 \\
\hline Tinca tinca & Tanche & 1,3 & 0,1 & 72,8 & 1,1 & 7 \\
\hline Leucaspius delineatus & Able de Heckel & 4,7 & 0,4 & 7,1 & 0,1 & 5 \\
\hline ESOX IUCHIS & Brochet & 1,0 & 0,1 & 40,0 & 0,6 & 5 \\
\hline Gobio gobio & Goujon & 0,7 & 0,1 & 4,2 & 0,1 & 4 \\
\hline Liza ramada & Mulet poro & 2,7 & 0,2 & 48,6 & 0,7 & 2 \\
\hline Carassius carassius & Carassin & 1,0 & 0,1 & 37,0 & 0,6 & 2 \\
\hline Atherina presbyter & Atherine & 0,5 & 0,0 & 1,4 & 0,0 & 2 \\
\hline Cyprinus carpio & Carpe commune & 0,3 & 0,0 & 240,0 & 3,7 & 2 \\
\hline Hippocampus ramulosus & Hippocampe & 0,3 & 0,0 & 1,7 & 0,0 & 2 \\
\hline Salmo salar & Saumon & 0,2 & 0,0 & 11,0 & 0,2 & 2 \\
\hline Syngnathus rostellatus & Syngnathe & 0,2 & 0,0 & 0,2 & 0,0 & 2 \\
\hline Pomatoschistus microos & Gobie tacheté & 0,2 & 0,0 & 0,0 & 0,0 & 2 \\
\hline Solea solea & Sole & 0,2 & 0,0 & 0,0 & 0,0 & 2 \\
\hline Abondances totales & & 1248,3 & & 6566,5 & & \\
\hline
\end{tabular}

L'observation des tailles des différentes espèces capturées (Tableau 3) indique que les espèces marines sont essentiellement représentées par les stades biologiques les plus jeunes (première et seconde années de vie), alors que les espèces résidentes de la baie et les espèces dulçaquicoles sont représentées par des juvéniles et quelques adultes. 


\section{Tableau III}

Tailles moyennes, minimales et maximales (en $\mathrm{mm}$ ) des différentes espèces piscicoles échantillonnées dans l'estuaire du Couesnon. $n$ : nombre d'îndividus mesurés. Classement par ordre alphabétique des espèces.

\section{Table III}

Fork length mean, min. and max. $(\mathrm{mm})$ of different fish species sampled in Couesnon estuary. $n$ : number of measured fish. Species are listed in alphabetic order.

\begin{tabular}{lrrrr}
\hline Espèce & \multicolumn{3}{c}{ Taille $(\mathrm{mm})$} & $\mathrm{n}$ \\
minimale & maximale & \\
\hline Able de Heckel & 61 & 44 & 92 & 14 \\
Ablette & 110 & 25 & 140 & 139 \\
Anguille & 282 & 66 & 840 & 31 \\
Athérine & 64 & 45 & 83 & 2 \\
Bar & 49 & 27 & 176 & 454 \\
Brème bordelière & 77 & 40 & 164 & 90 \\
Brème commune & 98 & 20 & 440 & 346 \\
Brochet & 169 & 64 & 254 & 3 \\
Carassin & 120 & 115 & 128 & 3 \\
Carpe commune & 585 & 585 & 585 & 1 \\
Chevaine & 91 & 34 & 415 & 333 \\
Epinoche & 45 & 18 & 70 & 48 \\
Flet & 82 & 18 & 336 & 159 \\
Gardon & 78 & 27 & 170 & 438 \\
Gobie buhotte & 40 & 16 & 69 & 1037 \\
Gobie de Lozanoi & 50 & 30 & 69 & 121 \\
Gobie tacheté & 35 & 35 & 35 & 1 \\
Goujon & 71 & 65 & 76 & 2 \\
Hippocampe & 100 & 100 & 100 & 1 \\
Mulet porc & 131 & 100 & 425 & 85 \\
Mulet spp. (alevins) & 65 & 28 & 100 & 338 \\
Plie & 57 & 16 & 370 & 616 \\
Rotengle & 102 & 70 & 149 & 13 \\
Saumon & 160 & 160 & 160 & 1 \\
Sole & 8 & 8 & 8 & 1 \\
Sprat & 39 & 28 & 49 & 114 \\
Syngnathe & 135 & 135 & 135 & 1 \\
Tanche & 154 & 29 & 490 & 4 \\
& & & & \\
\hline & & & &
\end{tabular}

\section{Variations spatio-temporelle}

Les différentes populations présentes varient en fonction des stations et des saisons. Les analyses hiérarchiques des correspondances sur les indices d'abondance des populations capturées dans les différentes stations durant les quatre saisons permettent de remarquer que la distribution spatiale est plus discriminante que la distribution temporelle (Figures 2 et 3 ). En effet que ce soit pour les indices d'abondance numérique ou pondérale, la première division sépare les échantillonnages des stations aval (stations 1 à 3 ) de ceux des stations amont (stations 4 à 6 ; groupe $\mathrm{C}$ ) en deux groupes. Dans le premier groupe, la seconde division (plus de $50 \%$ de dissimilarité pour 
les effectifs et plus de $60 \%$ pour les biomasses) sépare tous les échantillonnages effectués au niveau de la station 1 (groupe A) des autres stations aval (groupe B). Dans le groupe $C$, à l'exception de la station 1, se trouvent aussi tous les échantillonnages effectués en hiver où, comme pour les stations amont, les indices d'abondance et la richesse spécifique sont minimaux. La distribution spatiale s'observe surtout au niveau des biomasses des stations amont (groupe $C$ ) où trois sous groupes sont séparés : (1) les échantillonnages effectués en hiver, (2) en automne, et (3) au printemps et en été.

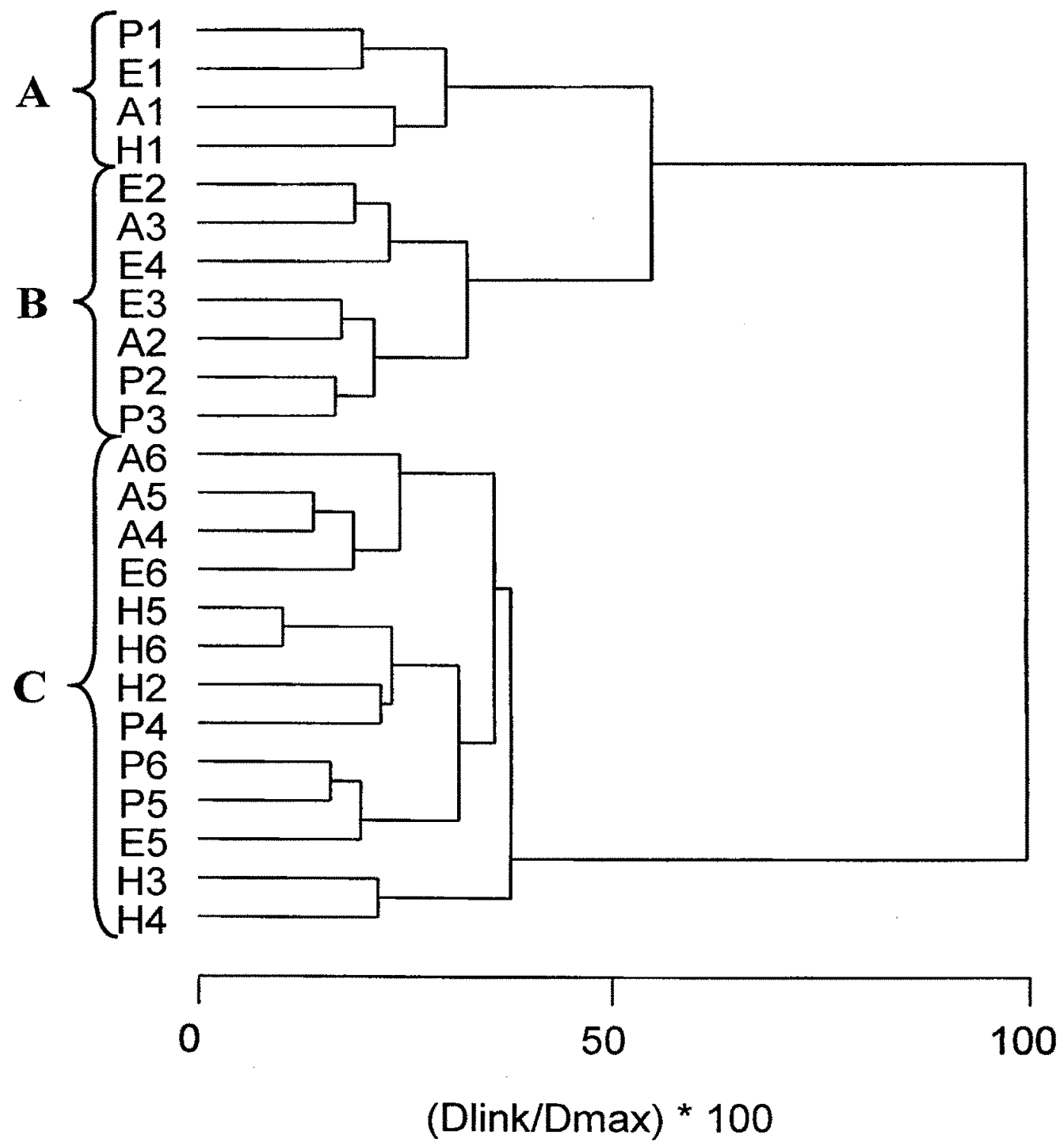

Figure 2

Classification hiérarchique (méthode de Ward, distance euclidienne) des stations en fonction des indices d'abondance numérique des espèces piscicoles échantillonnées. La distance des liens (Dlink) est représentée comme le pourcentage de la distance maximale des liens (Dmax). $\mathbf{P}$ : printemps, $\mathbf{E}$ : été, A : automne, $H$ : hiver. 1 à 6 : numéro des stations échantillonnées.

\section{Figure 2}

Cluster analysis (Ward's method, euclidean distances) on study sites in function densities index of species caught. The linkage distance (Dlink) is presented as a percentage of the maximum linkage distance (Dmax). $P$ : spring, $E$ : summer, $A$ : autumn, $H$ : winter. 1 to 6 : number of sampling sites. 


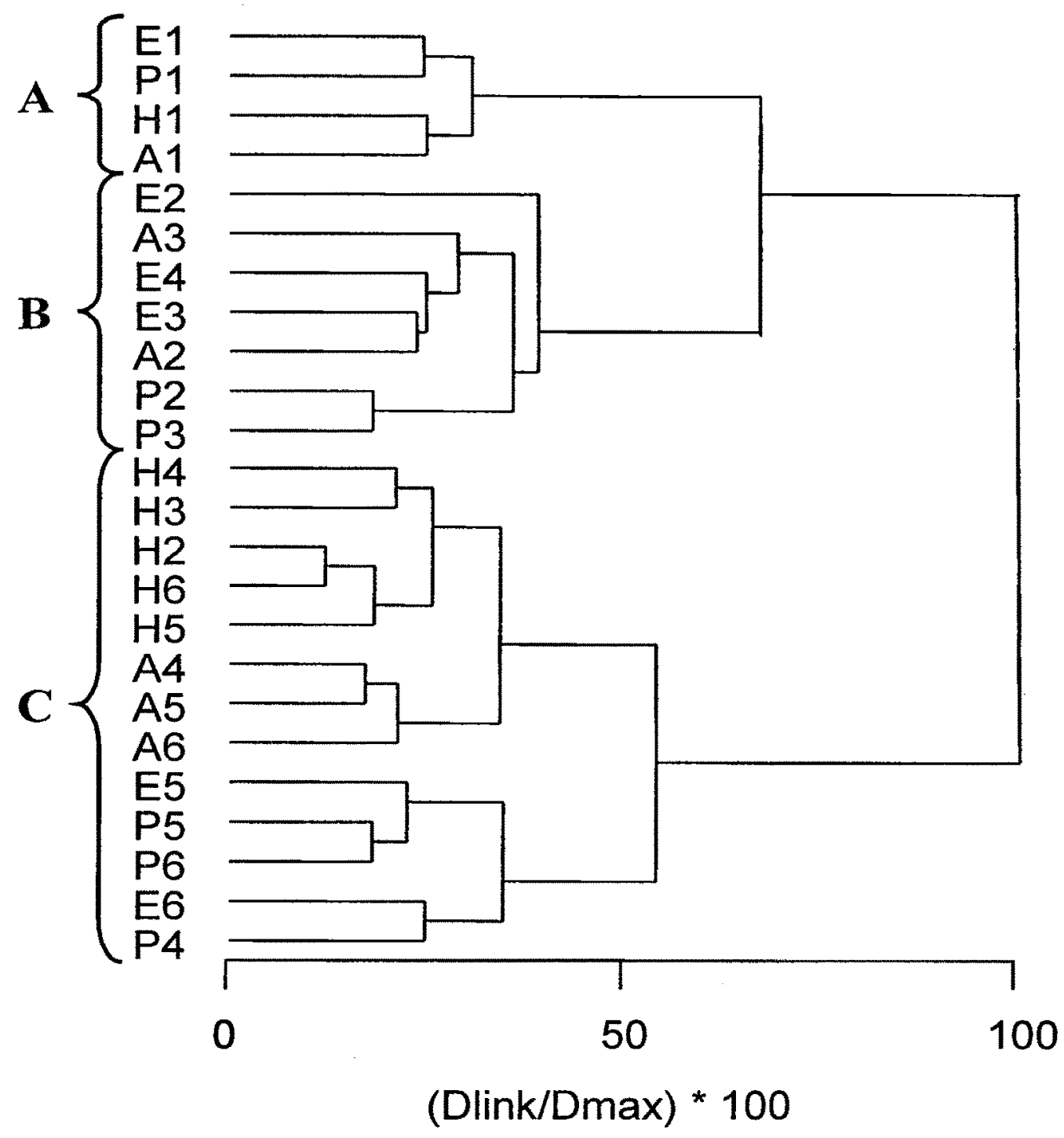

Figure 3

Classification hiérarchique (méthode de Ward, distance euclidienne) des stations en fonction des indices d'abondance pondérale des espèces piscicoles échantillonnées. La distance des liens (Dlink) est représentée comme le pourcentage de la distance maximale des liens (Dmax). P : printemps, $E$ : été, A : automne, $\mathrm{H}$ : hiver. 1 à 6 : numéro des stations échantillonnées.

Figure 3

Cluster analysis (Ward's method, euclidean distances) on study sites in function abundance index of species biomass caught. The linkage distance (Dlink) is presented as a percentage of the maximum linkage distance (Dmax). P : spring, $E$ : summer, $A$ : autumn, $H$ : winter. 1 to 6 : number of sampling sites. 


\section{Tableau IV}

Indice d'abondance moyenne des effectifs ( $\mathrm{nb} / \mathrm{ha}$ ) de l'ichtyofaune capturée dans les six stations de l'estuaire du Couesnon. Classement des espèces en fonction de leur répartition longitudinale.

\section{Table IV}

Mean density abundance index ( $\mathrm{nb} / \mathrm{ha}$ ) and species richness of fish community caught in six stations of Couesnon estuary. Species are listed according to their. spatial repartition.

\begin{tabular}{|c|c|c|c|c|c|c|}
\hline \multirow[t]{2}{*}{ Espèces } & \multicolumn{6}{|c|}{ Stations } \\
\hline & 1 & 2 & 3 & 4 & 5 & 6 \\
\hline Sole & 1 & - & - & - & - & - \\
\hline Syngnathe & 1 & - & - & - & - & - \\
\hline Saumon & 1 & - & - & - & - & - \\
\hline Athérine & 3 & - & - & - & - & - \\
\hline Gobie tacheté & - & 1 & - & - & - & - \\
\hline Hippocampe & - & 2 & - & - & - & - \\
\hline Sprat & 129 & 4 & - & - & - & - \\
\hline Gobie de Lozanoi & 157 & - & - & 2 & - & - \\
\hline Bar & 103 & 430 & 272 & $2 \overline{6}$ & - & - \\
\hline Alevin de mulet & 398 & 96 & 94 & - & 2 & 2 \\
\hline Plie & 162 & 470 & 316 & 186 & - & 5 \\
\hline Gobie buhotte & 758 & 540 & 116 & 28 & - & 18 \\
\hline Epinoche & 23 & 6 & - & 6 & 28 & 10 \\
\hline Anguille & 16 & 8 & 12 & 6 & 4 & 6 \\
\hline Flet & 13 & 178 & 86 & 90 & - & 1 \\
\hline Chevaine & 1 & 210 & 252 & 88 & 38 & 55 \\
\hline Gardon & 2 & 90 & 176 & 220 & 282 & 63 \\
\hline Brème bordelière & 3 & 102 & 10 & 16 & 8 & 27 \\
\hline Brème commune & 1 & 310 & 102 & 82 & 78 & 91 \\
\hline Able de Heckel & - & 22 & - & 6 & - & - \\
\hline Ablette & - & 78 & 36 & 54 & 52 & 54 \\
\hline Carassin & - & 6 & - & - & - & - \\
\hline Goujon & - & - & 2 & 2 & - & - \\
\hline Mulet porc & - & - & 16 & - & - & - \\
\hline Carpe commune & - & - & - & 2 & - & - \\
\hline Rotengle & - & - & - & - & 8 & 14 \\
\hline Tanche & - & - & - & - & 6 & 2 \\
\hline Brochet & - & - & - & - & 4 & 2 \\
\hline Densité totale (nb/ha) & 1773 & 2553 & 1490 & 814 & 510 & 350 \\
\hline Biomasse totale $(\mathrm{kg} / \mathrm{ha})$ & 3.5 & 13.5 & 8.0 & 7.0 & 4.3 & 3.8 \\
\hline Nombre d'espèce & 17 & 17 & 13 & 15 & 11 & 14 \\
\hline
\end{tabular}

Dans les stations les plus en aval (station 1 et 2) ont été échantillonnées essentiellement des espèces marines et résidentes des vasières tidales de la baie (Tableau 4). On trouve dans ces stations à la fois des espèces fourrage telle que le gobie buhotte (indices d'abondance compris entre 540 et 758 individus et entre 0.4 et $0.5 \mathrm{~kg}$ par hectare) et des espèces d'importance halieutique telles que la plie (entre 162 et 470 individus et entre 0.3 et $0.9 \mathrm{~kg}$ par hectare), le bar (entre 103 et 430 individus et entre 0.1 et $0.5 \mathrm{~kg}$ par hectare) et les alevins de mulets (entre 96 et 398 individus et entre 0.3 et $1.4 \mathrm{~kg}$ par hectare). Ces espèces disparaissent du peuplement à partir de la station 5 . Les 
espèces dulçaquicoles sont abondantes dès la station 2 avec des indices d'abondance variant entre 78 et 310 individus (représentant 1.0 à $4.1 \mathrm{~kg}$ ) par hectare pour la brème commune, entre 63 et 282 individus (représentant 0.4 à $1.7 \mathrm{~kg}$ ) par hectare pour le gardon et entre 38 et 252 individus (représentant 0.4 à $2.8 \mathrm{~kg}$ ) par hectare pour le chevaine. Par conséquent les indices d'abondance sont maximaux au niveau de la station 2 (près de 2500 individus et $13.5 \mathrm{~kg}$ par hectare en moyenne sur l'année). Ces indices diminuent vers l'amont pour atteindre leur valeur minimale à la station 6 (350 individus et $3.8 \mathrm{~kg}$ par hectare en moyenne sur l'année). Mais c'est au niveau de la station 1 que l'indice d'abondance pondéral est minimal ( $3.5 \mathrm{~kg}$ par hectare) à cause de la dominance presque exclusive de petites espèces (gobies), et des alevins et juvéniles d'origine marine (mulets, sprats, bars, plies).

À côté de cette distribution spatiale du peuplement piscicole, une évolution saisonnière peut aussi être observée. Sur les 21 espèces échantillonnées au printemps, ce sont les plies ( $\% \mathrm{~N}=33 \%)$ et les gardons $(\% \mathrm{~N}=21 \%)$ qui dominent le peuplement dont l'indice d'abondance numérique total est d'environ 1400 individus par hectare. Pour les biomasses (indice d'abondance pondéral total $=8.1 \mathrm{~kg}$ ), ce sont les espèces dulçaquicoles qui dominent : les gardons $(\% \mathrm{~B}=21 \%)$, les brèmes communes $(\% \mathrm{~B}=21 \%)$ et les chevaines $(\% \mathrm{~B}=18 \%)$. C'est lors de la campagne d'été que les indices d'abondance totale (près de 2200 poissons et $10.7 \mathrm{~kg}$ par hectare) et la diversité spécifique (22 espèces capturées) sont les plus élevées. Les espèces marines sont les plus abondantes numériquement ( $\% \mathrm{~N}=23 \%$ pour les jeunes bars et $16 \%$ pour les gobies buhottes) alors que ce sont toujours les espèces dulçaquicoles qui sont les plus abondantes pondéralement $(\% \mathrm{~B}=26 \%$ pour les chevaines et $19 \%$ pour les brèmes communes). En automne les indices d'abondance totale sont intermédiaires ( 1000 individus et $5.0 \mathrm{~kg}$ par hectare). Parmi les 16 espèces capturées, trois dominent numériquement le peuplement : les gobies buhottes $(\% \mathrm{~N}=29 \%)$, les alevins de mulets $(\% \mathrm{~N}=14 \%)$ et les brèmes communes $(\% \mathrm{~N}=12 \%)$. Concernant les biomasses, ce sont les brèmes communes $(\% \mathrm{~B}=31 \%)$, les anguilles $(\% \mathrm{~B}=13 \%)$ et les flets $(\% \mathrm{~B}=12 \%)$ qui sont les plus abondants. En hiver seulement 14 espèces ont pu être échantillonnées pour des indices d'abondance totale de 450 individus et $2.4 \mathrm{~kg}$ par hectare. Les gobies buhottes $(\% \mathrm{~N}=59 \%)$ et les carpes communes $(\% \mathrm{~B}=45 \%)$ sont les plus abondants.

\section{DISCUSSION}

\section{Peuplement général}

La pêche électrique est l'engin de pêche le plus fiable pour caractériser la structure et l'abondance du peuplement dans les zones dulçaquicoles (salinité $<1 \%$ ) de faible profondeur $(<1 \mathrm{~m}$ ) (FEUNTEUN, 1994 ; LAMBERT et al., 1994). Elle n'a pas pu être utilisée dans cet estuaire sous influence maritime. Dans les zones saumâtres, les engins de capture les plus utilisés sont le verveux ou le chalut (COSTA, 1986 ; POTTER et al., 1986 ; MAES et al, 1997). D'autres auteurs ont utilisé des sennes de plage (LE MAO, 1986 ; CLARK et al., 1996 ; JELLYMAN et al., 1997). Mais ces différentes méthodes ne permettent pas d'estimer les densités des différentes populations. Lors de cette étude, deux engins de pêche aux sélectivités complémentaires ont été utilisés. La senne de plage permet surtout de capturer le necton benthique alors que le filet maillant est plus efficace vis-à-vis de plus grands individus pélagiques. Le couplage de ces deux engins permet donc d'augmenter les gammes de taille et les zones échantillonnées. Toutefois, cette méthode ne donne que des indices d'abondance, ce qui est équivalent à un passage unique en pêche électrique. Le problème de cette technique composite est que certaines espèces, telles que l'anguille, ou écophases, tels que les « adultes " de poissons plats, sont sous représentées à cause de leur importante capacité d'échappement vis-à-vis de ces deux engins de pêche. 
Le peuplement piscicole échantillonné dans la zone estuarienne du Couesnon est assez typique des estuaires européens (CLARIDGE et al, 1986 ; POTTER et al., 1986 ; ELLIOT et DEWAILLY, 1995 ; MAES et al., 1998). Mais comparativement, peu d'espèces ont été recensées (28 espèces), même si la diversité spécifique est en partie déterminée par l'effort de pêche (ALLEN et al., 1992). En effet, dans 17 estuaires européens, la diversité spécifique varie entre 22 et 94 espèces (ELLIOT et DEWAILLY, 1995). Cette relative faible diversité spécifique est aussi certainement due à la petite taille de l'estuaire. Le peuplement de l'estuaire du Couesnon est dominé par 7 espèces : 1 provenant des vasières tidales (le gobie buhotte), 3 d'origine marine (la plie, le bar et les alevins de mulet) et 3 d'origine dulçaquicole (le gardon, la brème commune et le chevaine) représentant près de $80 \%$ des effectifs et $70 \%$ des biomasses totaux capturés. Les gobies du genre Pomatoschistus sont connus pour dominer les peuplements littoraux de l'ouest européen (HAMERLYNCK et al., 1993a ; HAMERLYNCK et CATTRIJSSE, 1994 ; COSTA et CABRAL, 1999). Accompagnés des plies, des bars et des mulets, ce sont aussi les plus communes et les plus abondantes des vasières (LEGENDRE, 1984 ; FEUNTEUN et LAFFAILLE 1997) et des marais salés tidaux (LAFFAILLE et al., $1998 ; 2000 a$ ) de la baie du Mont Saint-Michel. Les trois cyprinidés sont typiques des zones aval des cours d'eau français ou zones à brèmes (HUET, 1949 ; 1959 ; VERNEAUX, 1977). D'autres travaux confirment la position dominante d'un petit nombre d'espèces dans les communautés estuariennes (CLARIDGE et al., 1986 ; ELLIOT et TAYLOR, 1989 ; HENDERSON, 1989 ; MAES et al., 1997).

Les structures de taille montrent pour toutes les espèces marines une nette dominance des individus de la première et de la seconde année de vie, comme cela a déjà été observé dans de nombreuses lagunes côtières et estuaires des régions tempérées (DAHLBERG et ODUM, 1970 ; CAIN et DEAN, 1976 ; MARCHAND, 1980 ; BECKLEY, 1984 ; QUIGNARD et al., 1984 ; POTTER et al., 1986). Mais les structures de ces communautés sont certainement affectées par l'absence présumée de grands individus par rapport aux juvéniles (DAY et al., 1989). Les espèces marines qui entrent dans les estuaires durant leur première année de vie, utilisent généralement ces zones uniquement comme des nurseries (par exemple ici le bar et la plie) (LEMANTON et POTTER, 1987). Les juvéniles de ces espèces utilisent certainement les transports tidaux passifs et sélectifs pour arriver dans les estuaires (FORTIER et LEGGETT 1982 ; NORCROSS et SHAW 1984 ; APRAHAMIAN et BARR 1985 ; DAME et ALLEN, 1996). Les petits estuaires sont des milieux peu profonds et instables. De telles conditions excluent un grand nombre d'espèces dont les grands prédateurs. Ce sont alors des zones de protection pour les plus jeunes poissons, stades critiques où s'opèrent un grand nombre de facteurs affectant par la suite les stocks de poissons exploités (SMITH, 1985). Toutefois, quelques espèces peuvent aussi exploiter périodiquement les estuaires au stade adulte (par exemple les mulets). Ce groupe d'espèces marine peut dominer numériquement les taxa présents et constituer plus de $70 \%$ du necton (WEINSTEIN $1979 ; 1981$ ). Les véritables espèces estuariennes peuvent accomplir entièrement leur cycle biologique dans ces milieux (DANDO, 1984) qui représentent alors un habitat permanent pour tous les stades. Beaucoup sont des espèces fourrages comme les gobies du genre Pomatoschistus qui jouent un rôle important dans la dynamique trophique de ces systèmes (WEINSTEIN, 1982). Des espèces dulçaquicoles les plus euryhalines (comme les brèmes, les chevaines et les gardons) peuvent aussi habiter les estuaires, comme un petit nombre d'espèces marines qui restent surtout dans les zones saumâtres (POTTER et al., 1986 ; 1990). Enfin, pour les espèces diadromes, telles que l'anguille ou les salmonidés, les estuaires sont un passage obligatoire entre les milieux dulçaquicoles et marins (Mc DOWALL, 1988).

\section{Variation spatiale}

Spatialement, les communautés piscicoles des estuaires européens sont fortement organisées et largement structurées par la salinité et le substrat (KEUP et BAYLESS, 1964 ; MILLS, 1975 ; HENDERSON, 1989 ; HAMERLYNCK et al., 1993b ; MAES et al., 1998). Secondairement, la composition spécifique peut être influencée par les 
caractéristiques géomorphologiques (surface, proportion d'eau courante et calme, etc.), hydrodynamiques (courant, amplitudes des marées, etc.), des paramètres abiotiques (température, oxygène, etc.) et des statuts trophiques (nutriment, densité en plancton, etc.) (COPELAND et BECHTEL, 1974 ; NEILL et CULLEN, 1974 ; WEINSTEN et WALTERS, 1981 ; WEISTEIN, 1982 ; THIEL et al., 1995). La présence de poissons et leur répartition spatiale dans les estuaires peut donc dépendre de nombreux facteurs abiotiques et biotiques qui agissent souvent en synergie. De plus, la tolérance aux variations de ces différents paramètres est différente non seulement selon les espèces mais aussi selon les stades biologiques d'une même espèce. Pour l'estuaire du Couesnon, la salinité est certainement le facteur principal dans la répartition longitudinale des populations piscicoles. REMANE et SCHLIEPER (1971) définissent une relation positive entre la salinité et le nombre d'espèces. Cette relation peut être observée dans l'estuaire du Couesnon où les stations les plus en aval (stations 1 et 2) de l'estuaire possèdent les indices cie diversité et les indices d'abondance piscicoles les plus élevés. Un résultat identique a été trouvé dans l'estuaire du Tage (Portugal) par COSTA (1989). Les importants taux de salinité dans ces zones, proches des valeurs du domaine maritime, permettent la présence de nombreuses espèces marines côtières qui s'y nourrissent.

Dans l'estuaire du Couesnon, ce gradient est accentué par deux aménagements hydrauliques : le barrage estuarien et la banalisation des berges. L'effet barrage le plus évident est de faire obstacle aux déplacements génésiques et/ou trophiques des migrateurs et en particulier pour les espèces amphihalines. Cet obstacle a un effet important sur la structure des peuplements : it concentre en amont ou en aval (en fonction du sens des migrations) les populations migrantes (ELIE et al., 1990 ; ROUX et COPP, 1993 ; FEUNTEUN et al., 1998). Le barrage estuarien limite les migrations trophiques et génésiques de la plupart des espèces diadromes et d'origine marine. De plus, ce barrage modifie, et lors de certaines saisons interrompt, le gradient de salinité. Cet effet sur le paramètre abiotique primordial de la structuration spatiale des peuplements piscicoles explique certainement la faible diversité spécifique relative de cet estuaire par rapport aux autres estuaires européens. De plus, le gradient de salinité se déplace entre l'amont et l'aval en fonction du débit, ce qui peut expliquer la remontée des espèces euryhalines marines vers l'amont en été en suivant le front salin et le déplacement des espèces euryhalines dulçaquicoles vers l'aval en hiver avec l'augmentation du débit. La banalisation et la chenalisation des berges réduisent la diversité et la quantité des habitats disponibles. Or celles-ci peuvent procurer à l'état naturel des zones de frayères et de croissance (SCHIEMER et SPINDLER, 1989 ; ROUX et COPP, 1993). PONT et PERSAT (1990) ont trouvé une relation positive hautement significative entre le rapport longueur totale des rives sur longueur linéaire du cours d'eau et l'abondance moyenne en poissons. Cette augmentation d'abondance et très souvent due à la présence d'un grand nombre de jeunes de l'année.

\section{Variation temporelle}

Comme lors de l'étude de MAES et al. (1997) nous n'avons pas pu observer clairement de variabilité saisonnière de la composition piscicole, à l'exception des zones amont pour les biomasses. Or, de nombreux auteurs ont identifié des variations saisonnières de la composition, des indices d'abondance et de la diversité des poissons et des crustacés des estuaires (DAHLBERG et ODUM, 1970 ; LIVINGSTON 1976 ; QUINN 1980 ; WHARFE et al., 1984 ; CLARIDGE et al., 1986 ; HENDERSON et HOLMES, 1987). Les différentes analyses montrent que la distribution des communautés est beaucoup plus structurée longitudinalement que temporellement. D'autres auteurs ont souligné que les variations temporelles des communautés piscicoles et les facteurs qui contrôlent ces variations peuvent être visualisés hiérarchiquement que ce soit dans les zones côtières (ROSS et al., 1987) ou les estuaires (CLARIDGE et al., 1986 ; POTTER et al., 1986). En effet, ce sont les événements climatiques qui influencent les variations annuelles, les mouvements trophiques et génésiques qui influencent les variations saisonnières, alors que les variations à court terme semblent influencées par des facteurs physico-chimiques comme la salinité, la température et les taux d'oxygène dissous. 
Dans les nurseries estuariennes européennes, le recrutement débute typiquement en avril et juin. Après un maximum des diversités spécifiques et des indices d'abondance durant l'été, les effectifs déclinent à cause de la mortalité naturelle due essentiellement à la prédation ou au départ du système et ceci jusqu'en automne (septembre à octobre) où les survivants migrent vers les eaux plus profondes. La nature migratrice, au sens large, de nombreuses espèces est une des caractéristiques des populations piscicoles estuariennes qui influence directement les indices de diversité et les indices d'abondance saisonniers (CAIN et DEAN, 1976).

\section{CONCLUSION}

Cette étude sur la répartition spatio-temporelle des communautés piscicoles de l'estuaire du Couesnon a permis de délimiter trois grandes zones d'aval en amont. Une zone estuarienne marine (correspond au secteur 1) composée principalement d'espèces marines euryhalines et résidentes des vasières de la baie. La diversité et les densités des espèces présentes y sont importantes et proches de celles des vasières et des marais salés tidaux adjacents. La fonction trophique et de nurserie jouée par cette zone est elle aussi proche de celle des marais salés (LAFFAILLE et al., 1998 ; 1999 ; 2000b). La troisième zone est une zone estuarienne dulçaquicole (correspondant aux secteurs 4 à 6 ) où le peuplement est constitué presque exclusivement d'espèces dulçaquicoles caractéristiques des zones à brèmes, avec des densités relativement faibles. Ces deux zones sont relativement stables aux cours des saisons. La seconde zone (secteurs 2 et 3 ) correspond à une zone intermédiaire, est constituée d'espèces marines euryhalines et résidentes et d'espèces dulçaquicoles durant le printemps et l'été. En automne et en hiver, l'influence de la salinité en amont du barrage estuarien est faible permettant aux espèces dulçaquicoles de coloniser cette zone. La présence du barrage estuarien réduit donc dans l'espace et le temps le rôle de nurserie vis-à-vis des espèces marines euryhalines telles que le bar, la plie et les mulets. La banalisation et la chenalisation des berges sont aussi deux facteurs incriminés dans les faibles densités et diversités de cet hydrosystème. Or, les estuaires tidaux sont d'une grande importance tant pour les poissons côtiers que pour ceux des zones aval des rivières (ROZAS et ODUM, 1987). De plus, ils contribuent significativement à la biodiversité de l'écosystème baie du Mont Saint-Michel dans son entier et à l'enrichissement halieutique de la baie.

\section{REMERCIEMENTS}

Cette étude a été financée par le Syndicat Mixte pour rétablissement du caractère maritime du Mont Saint-Michel et la Mission du Mont Saint-Michel (Direction Départementale de l'Equipement de la Manche). Les auteurs remercient les nombreux collègues pour leur aide indispensable et plus particulièrement Calou, T. Robinet et A. Radureau.

\section{BIBLIOGRAPHIE}

ALLEN D.M., SERVICE S.K., OGBURN-MATTHEWS M.V., 1992. Factors influencing the collection efficiency of estuarine fishes. T. Am. Fish. Soc., 121, 234-244.

APRAHAMIAN M.W., BARR C.D., 1985. The growth, abundance and diet of 0-group sea bass, Dicentrarchus labrax, from the Severn Estuary. J. Mar. Biol. Ass. U.K., 65, 169-180.

BECKLEY L.E., 1984. The ichtyofauna of the Sundays Estuary, South Africa, with particular reference to the juvenile marine component. Estuaries, 7, 248-258. 
BLABER S.J., BLABER T.G., 1980. Factors affecting the distribution of juvenile estuarine and inshore fish. J. Fish Biol., 17, 143-162.

CAIN R.J., DEAN J.M., 1976. Annual occurrence, abundance and diversity of fish in a South Carolina intertidal creek. Marine Biology, 36, 369-379.

CLARK B.M., BENNETT B.A., LAMBERTH S.J., 1996. Temporal variations in surf zone fish assemblages from False Bay, South Africa. Mar. Ecol. Prog. Ser., 131, 35-47.

CLARIDGE P.N., POTTER I.C., 1984. Abundance, movements and size of gadoids (Teleostei) in the Severn Estuary. J. Mar. Biol. Ass. U.K., 64, 771-790.

CLARIDGE P.N., POTTER I.C., HARDISTY M.W., 1986. Seasonal changes in movements, abundance, size composition and diversity on the fish fauna of the Severn Estuary. J. Mar. Biol. Ass. U.K., 66, 229-258.

COPELAND B.J., BECHTEL T.J., 1974. Some environmental limits of six gulf coast estuarine organisms. Contrib. Mar. Sci., 18, 169-204.

COSTA M.J., 1986. Les poissons de l'estuaire du Tage. Cybium, 10, 57-75.

COSTA M.J., 1988. The Tagus and Mira estuaries (Portugal) and their role as spawning and nursery areas. J. Fish Biol., 33, 249-250.

COSTA M.J., 1989. The structure of fish communities in the Tagus Estuary, Portugal, and its role es a nursery for commercial fish species. Scient. Mar., 53, 561-566.

COSTA M.J., CABRAL H.N., 1999. Changes in the Tagus nursery function for commercial fish species: some perspectives for management. Aquatic Ecology, 33, 287-292.

DAHLBERG M.D., ODUM E.P., 1970. Annual cycles of species occurrence, abundance, and diversity in Georgia estuarine fish populations. Am. Mid. Nat., 83, 382-392.

DAME R.F., ALLEN D.M., 1996. Between estuaries and the sea. J. Exp. Mar. Biol. Ecol., 200, 169-185.

DANAIS M., FOUILLET P., LEGENDRE C., 1987. Le Couesnon et les marais de sa basse vallée. Etat initial. Rapport Min. Equip. Laboratoire Evolution des Systèmes Naturels et Modifiés, Université de Rennes 1, $47 \mathrm{p}$.

DANDO P.R., 1984. Reproduction in estuarine fish in POTTS G.W., WOOTON R.J., Fish reproduction strategies and tactics, 155-170, Academic Press, London.

DAY J.W., HALL C.A.S., KEMP W.M., YANEZ-ARANCIBIA A., 1989. Estuarine ecology. Wiley, New York.

ELIE P., FEUNTEUN E., RIGAUD C., 1990. The inshore brackish water domain of the French Atlantic coast : ecological functions for the exploited species-impact of physical development. Bull. Ecol., 21, 33-38.

ELLIOTT M., DEWAILLY F., 1995. The structure and components of European estuarine fish assemblage. Neth. J. Aquat. Ecol., 29, 397-417.

ELLIOTT M., TAYLOR C.L.J., 1989. The structure and functioning of an estuarine/marine fish community in the Forth estuary, Scotland. Proceeding of the $21^{\text {st }}$ European Marine Biology Symposium, Gdansk, 227-240.

FEUNTEUN E., 1994. Le peuplement piscicole du marais littoral endigué de BourgneufMachecoul (France, Loire-Atlantique). Approche méthodologique pour une analyse quantitative de la distribution spatiale du peuplement et de la dynamique de certaine populations. Thèse de l'Université de Rennes 1, $250 \mathrm{p}$.

FEUNTEUN E., LAFFAILLE P., 1997. Les peuplements piscicoles de la baie du Mont Saint-Michel. Penn Ar Bed, 164, 50-56.

FEUNTEUN E., ACOU A., GUILLOUËT J., LAFFAILLE P., LEGAULT A., 1998. Spatial distribution of en eel population (Anguilla anguilla L.) in a small coastal catchment of northern Brittany (France). Consequences of hydraulic works. Bull. Fr. Pêche Piscic., 349, 129-139. 
FIELDS J.G., CLARKE K.R., WARWICK R.M., 1982. A practical strategy for analysing multispecies distribution patterns. Mar. Ecol. Prog. Ser., 8, 37-52.

FORTIER L., LEGGETT W.C., 1982. Fickian transport and the dispersal of fish larvae in estuaries. Can. J. Fish Aquat. Sci., 39, 1150-1163.

HAMERLYNCK O., CATTRIJSSE A., 1994. The food of Pomatoschistus minutus (Pisces, Gobiidae) in Belgian coastal waters, and a comparison with the food of its potential competitor P. lozanoi. J. Fish Biol., 44, 753-771.

HAMERLYNCK O., CATTRIJSSE A., ARELLANO R.V., 1993a. Daily ration of juvenile Pomatoschistus lozanoi de Buen (Pisces, Gobiidae). ICES J. Mar. Sci., 50, 471. 480.

HAMERLYNCK O., HOSTENS K., ARELLANO R.V., MEES J., VAN DAMME P.A., 1993b. The mobile epibenthic fauna of soft bottoms in the Dutch Delta (South West Netherlands) : spatial structure. Nert. J. Aquat. Ecol., 27, 343-358.

HENDERSON P.A., 1989. On the structure of the inshore fish community of England and Wales. J. Mar. Biol. Ass. U.K., 69, 145-163.

HENDERSON P.A., HOLMES R.H.A., 1987. On the population biology of the common shrimp Crangon crangon (L.) (Crustacea, Caridea) in the Severn estuary and Bristol Channel. J. Mar. Biol. Ass. U.K., 67, 825-847.

HUET M., 1949. Aperçu des relations entre la pente et les populations piscicoles des eaux courantes. Schweiz. Z. Hydrol., 11, 333-351.

HUET M., 1959. Profiles and biology of Western European streams as related to fish management. Trans. Am. Fish. Soc., 88, 155-163.

JELLYMAN D.J., GLOVA G.J., SAGAR P.M., SYKES J.R.E., 1997. Spatio-temporal distribution of fish in the Kakanui River estuary, South Island, New Zealand. New Zeal. J. Mar. Fresh., 31, 103-118.

KEUP L., BAYLESS J., 1964. Fish distribution in varying salinities in the Neuse River basin, North Carolina. Chesapeake Sci., 5, 119-123.

LAFFAILLE P., BROSSE S., FEUNTEUN E., BAISEZ A., LEFEUVRE J.-C., 1998. Role of fish communities in organic matter fluxes between salt marshes and coastal marine waters in the Mont Saint-Michel bay. Hydrobiologia, 373/374, 121-133.

LAFFAILLE P., FEUNTEUN E., LEFEUVRE J.C., 1999. Compétition alimentaire entre deux espèces de gobies, Pomatoschistus lozanoi (de Buen) et $P$. minutus (Pallas), dans un marais salé macrotidal. C.R. Acad. Sci. Paris, Life Sciences, 322, 897-906.

LAFFAILLE P., FEUNTEUN E., LEFEUVRE J.C., 2000a. Composition of fish communities in an European macrotidal salt marshes (the Mont Saint-Michel Bay, France). Estuar., Coast. Shelf S. (sous presse).

LAFFAILLE P., LEFEUVRE J.C., FEUNTEUN E., 2000b. Impact of sheep grazing on juvenile sea bass, Dicentrarchus labrax L., in tidal salt marshes. Biol. Conserv., 96, 271-277.

LAMBERT P., FEUNTEUN E., RIGAUD C., 1994. Etude de l'anguille en marais d'eau douce. Première analyse des probabilités de captures. Bull. Fr. Pêche et Piscic., $335,111-122$.

LEGENDRE G., 1984. La pêche artisanale sur le domaine intertidal de la baie du Mont Saint-Michel. Rapport CEE et Min. Env. Laboratoire Evolution des Systèmes Naturels et Modifiés, Université de Rennes 1, $122 \mathrm{p}$.

LEMANTON R.C., POTTER I.C., 1987. Contribution of estuaries to commercial fisheries in temperate Western Australiasia and the concept of estuarine dependence. Estuaries, 10, 28-35.

LE MAO P., 1986. Feeding relationships between the benthic infauna and the dominant benthic fish of the Rance Estuary (France). J. Mar. Biol. Ass. U.K., 66, 391-401. 
LIVINGSTON R.J., 1976. Diurnal and seasonal fluctuations of organisms in a North Florida estuary. Estuar. Coast. Mar. Sci., 4, 373-400.

MAES J., TAILLIEU A., VAN DAMME P.A., OLLEVIER F., 1997. The composition of the fish and crustacean community of the Zeeschelde estuary (Belgium). Belg. J. Zool, 127, 47-55.

MAES J., VAN DAMME P.A., TAILLIEU A., OLLEVIER F., 1998. Fish communities along an oxygen-poor salinity gradient (Zeeschelde Estuary, Belgium). J. Fish Biol., 52, $534-546$.

MANN K.H., 1982. Ecology of coastal waters. University of California press, Los Angeles, $322 \mathrm{p}$.

MARCHAND J., 1980. Les vasières de la basse Loire : leur rôle de nourricerie pour l'ichtyofaune. Compétition trophique interspécifique. Actes du congrès FRAPEC "Aménagement et écologie de la Loire ", Tours, 187-214.

MC DOWALL R.M., 1988. Diadromy in fishes : migrations between marine and freshwater environments. Croom Helm, London, $308 \mathrm{p}$.

Mc LUSKY D.S., 1981. The estuarine ecosystem. Blackie \& Son Ltd, London, $150 \mathrm{p}$.

MILLS E.L., 1975. Benthic organisms and the structure of marine ecosystems. J. Fish. Res. Board Can., 32, 1657-1663.

NEILL S.R., CULLEN J.M., 1974. Experiments on whether schooling by their prey affects the hunting behaviour of cephalopods and fish predators. J. Zool., 172, 549-569.

NORSCROSS B.L., SHOW R.F., 1984. Oceanic and estuarine transport of fish eggs and larvae : a review. T. Am. Fish. Soc., 113, 153-165.

PONT D., PERSAT H., 1990. Spatial variability of fish community in major central European regulated river. Symposium on Flooplain Rivers, 9-11 April 1990, Baton Rouge, Louisiana, USA.

POTTER I.C., BECKLEY L.E., WHITFIELD A.K., LEMANTON R.C.J., 1990. Comparisons between the roles played by estuaries in the life cycles of fishes in temperate Western Australia and Southern Africa. Environ. Biol. Fish., 28, 143-178.

POTTER I.C., CLARIDGE P.N., WARWICK R.M., 1986. Consistency of seasonal changes in an estuarine fish assemblage. Mar. Ecol. Prog. Ser., 32, 217-228.

QUIGNARD J.P., MAN WAI R., VIANET R., 1984. Les poissons de l'étang de Mauguio (Hérault, France). Inventaire, structure du peuplement, croissance et polymorphisme des tailles. Vie Milieu, 34, 173-183.

QUINN N.J., 1980. Analysis of temporal changes in fish assemblages in Serpentine creek, Queensland. Env. Biol. Fish, 5, 117-133.

REMANE A., SCHLIEPER C., 1971. Biology of brackish water. Wiley-Interscience, New York, $372 \mathrm{p}$.

ROGERS S.G., TARGETT T.E., van SANT S.B., 1984. Fish-nursery use in Georgia saltmarsh estuaries: the influence of springtime freshwater conditions. T. Am. Fish. Soc., 113, 595-606.

ROSS S.T., MC MICHAEL R.H., RUPLE D.L., 1987. Seasonal and diel variation in the standing crop of fishes and macroinvertebrates from a Gulf of Mexico surf-zone. Estuar. Coast. Shelf. Sci., 25, 391-412.

ROUX A.L., COPP G.H., 1993. Peuplements de poissons in AMOROS C., PETTS G.E., Hydrosystèmes fluviaux, 151-166, Collection d'Ecologie, Masson, Paris.

ROZAS L.P., ODUM W.E., 1987. Use of tidal freshwater marshes by fishes and macrofaunal crustaceans along a marsh stream-order gradient. Estuaries, 10, 3643.

SCHIEMER F., SPINDLER T., 1989. Endangered fish species of the Danue River in Austria. Regulated Rivers, 4, 397-407. 
SMITH P.E., 1985. Year-class strength and survival of 0-group clupeoids. Can. J. Fish. Aquat. Sci., 42, 69-82.

THIEL R., SEPULVEDA A., KAFEMANN R., NELLEN W., 1995. Environmental factors as forces structuring the fish community of the Elbe estuary. J. Fish Biol., 46, 47-49.

VERNEAUX J., 1977. Biotypologie de l'écosystème " eau courante ". C.R. Acad. Sci. Paris, 284, 77-80.

WEINSTEIN M.P., 1979. Shallow marsh habitats as primary nurseries for fishes and shellfish, Cape Fear River, North Carolina. Fish. Bull., 77, 339-357.

WEINSTEIN M.P., 1981. Plankton productivity and the distribution of fishes on the southeastern U.S. continental shelf. Science, 214, 351-352.

WEINSTEIN M.P., 1982. Commentary : a need for more experimental work in estuarine fisheries ecology. Northeast Gulf Science, 5, 59-64.

WEINSTEIN M.P., WALTERS M.F., 1981. Growth, survival and production in young-of-theyear populations of Leiostomus xanthurus Lacepede, residing in tidal creeks. Estuaries, 4, 185-197.

WHARFE J.R., WILSON S.R., DINES R.A., 1984. Observations on the fish populations of an East Coast Estuary. Mar. Pollut. Bull., 15, 133-136.

WHITFIELD A.K., 1983. Factors influencing the utilization of southern African estuaries by fishes. S. Afr. J. Sci., 79, 362-365. 